\title{
Introduction of a New Journal
}

\author{
John M. Hallenbeck
}

Published online: 17 February 2010

(C) National Institutes of Health 2010

Both basic and clinical stroke research continue to deepen and expand our understanding of stroke pathobiology, but there remains a gap in the medical application of that understanding that has been remarkably difficult to bridge. Preclinical and clinical data generated from disciplines such as Pathology, Physiology, Biochemistry, Immunology, Molecular Biology, Cell Biology, Developmental Neuroscience, and nascent efforts in Systems Biology along with critical technical advances such as Neuroimaging provide a reasonably coherent and detailed picture of the sweep and complexity of stroke. Also, the available stroke-related bioinformation has guided some notable clinical successes. Nonetheless, there remains a gap in stroke research between the bench and the bedside that needs bridging. Although evolving recommendations such as STAIR Criteria continue to improve the design of animal stroke models, preclinical stroke data often do not permit accurate predictions of the likelihood that translation into the clinical arena of innovations in prevention, treatment, or repair of stroke will be successful. Further, it seems improbable that clinical trial designs, though they are still being optimized, will ever achieve a sensitivity and compression of variability that approaches the limit of detection for preclinical studies that can show small beneficial effects of interventions. This suggests the existence of a potency threshold for innovations

J. M. Hallenbeck ( $\square)$

NINDS, NIH,

Bethesda, MD, USA

e-mail: HallenbJ@ninds.nih.gov in stroke management below which successful translation is virtually impossible. On the basis of these and many other conundrums, go-no-go translation decisions can easily end up being misguided.

As more institutional stroke research programs shift from serial studies and serial communications of novel molecular mechanisms (done in the hope that the stroke field itself will steadily move toward improved stroke care) to a more accelerated multidisciplinary process of target discovery and preclinical/clinical development of promising innovations for stroke management, a journal dedicated to translation can provide a forum for all matters of interest to translational stroke researchers and for dissemination of ideas and solutions to problems as they arise. Regulatory issues can be examined, and strategies for streamlining preclinical and phase I/II development of candidate agents for management of stroke can be presented, discussed, debated, and refined. Pilot trials can be published and serve as positive or negative models for development of promising innovations. Translational research is inherently multidisciplinary, and a dedicated journal can foster increased interaction and communication among the diverse members of translational research teams. A journal dedicated to stroke translation can also serve as an organ for translational research in other stroke-related complex diseases such as traumatic brain injury.

With a stroke occurring somewhere in the world every few seconds on average and averaging once every $42-45 \mathrm{~s}$ in the USA alone, there is clearly a pressing unmet need to bridge this gap by improving the design and conduct of translational stroke research. This new journal, Translational Stroke Research, will serve as a platform to help support the bridging of this gap. 